\title{
Walking impairment in patients with multiple sclerosis: exercise training as a treatment option
}

\author{
This article was published in the following Dove Press journal: \\ Neuropsychiatric Disease and Treatment \\ 15 November 2010 \\ Number of times this article has been viewed
}

\author{
Robert W Motl' \\ Myla D Goldman ${ }^{2}$ \\ Ralph HB Benedict ${ }^{3}$ \\ 'Department of Kinesiology and \\ Community Health, University of \\ Illinois at Urbana-Champaign, Urbana, \\ IL, USA; ${ }^{2}$ Department of Neurology, \\ University of Virginia, VA, USA; \\ ${ }^{3}$ Department of Neurology, SUNY \\ Buffalo School of Medicine, Buffalo, \\ NY, USA
}

\begin{abstract}
Multiple sclerosis (MS) is a chronic disease of the central nervous system that culminates in the progression of physical and cognitive disability over time. Walking impairment is a ubiquitous feature of MS and a sentinel characteristic of the later or advanced stages of the disease. This paper presents a conceptual rationale along with empirical evidence for exercise training as a rehabilitation approach for managing walking impairment and improving walking function in persons with MS. Conceptually, MS is associated with a decrease in physical activity, which, in turn, can result in deconditioning across multiple domains of physiological functioning. The resulting deconditioning feeds back and further drives physical inactivity until a threshold is reached that likely initiates the progression of walking impairment in MS. Empirically, physical activity and exercise training have been associated with beneficial effects on walking function in persons with MS. This is based on cross-sectional, longitudinal, and experimental research that included diversity in the breadth of measures of walking, persons with MS, and exercise/ physical activity characteristics. Of particular importance, future researchers might consider examining the combinatory effects of exercise training plus pharmacological agents on walking mobility in MS. Collectively, exercise training and physical activity might hold significant potential for the management of progressive mobility disability in MS.
\end{abstract}

Keywords: mobility, walking, exercise, physical activity, movement

\section{Introduction}

Multiple sclerosis (MS) is a chronic disabling disease of the central nervous system (CNS) that affects approximately 1 per 1000 persons in the United States. ${ }^{1}$ The majority of persons are diagnosed between the ages of 20 and 50 years, and women are more often affected than men. ${ }^{2} \mathrm{MS}$ is more prevalent among people with northern European ancestry than those of African, Asian, and Hispanic ancestry. ${ }^{2}$ The most common course of MS is initially characterized by intermittent and recurrent episodes of multifocal inflammation in the CNS. ${ }^{3}$ Such inflammatory processes result in the demyelination and transection of axons in the brain, optic nerve, and spinal cord. ${ }^{4}$ The axonal damage results in conduction delay and block of electrical potentials along neuronal pathways. ${ }^{5}$ Over the course of MS, there are further neurodegenerative processes that are presumably characterized by insufficient support of nerve growth factors within the CNS. ${ }^{4,5}$ The inflammatory and neurodegenerative processes and associated injury culminate in the progression of physical and cognitive disability in persons living with MS.

Walking impairment is one of the most ubiquitous features of MS and is a sentinel characteristic of the later or advanced stages of the disease. Importantly, walking impairment has been seen early in the MS disease course ${ }^{6}$ and represents a particular
Correspondence: Robert W Mot

Department of Kinesiology and

Community Health, University

of Illinois, 906 S. Goodwin Ave,

Urbana, IL 6I80I, USA

Tel +l 2172650886

Fax +12173333124

Email robmotl@uiuc.edu 
concern of those living with this disease. The identification of therapeutic options for managing walking impairment and improving walking function in persons with MS therefore represents a topic of important scientific inquiry. This paper presents a conceptual rationale along with scientific evidence for exercise training as a rehabilitation approach for managing walking impairment and improving function in persons with MS. We have developed this rationale in four sections that offer important vantage points on the topic. The first section of the paper provides a background on the extent of impairment in walking among those with MS. The second section focuses on physiological deconditioning as a major contributing and alterable factor that underlies the progression of walking impairment over time in MS. This sets the background for the third section, which presents scientific evidence for exercise training and physical activity as therapeutic modalities for managing walking impairment in MS. The final section provides an agenda for consideration by researchers with a particular focus on exercise training along with pharmacological agents as a combinatory approach for managing impairment of walking in MS. The over-arching goal of this paper is the provision of a scholarly resource for clinicians and practitioners who are interested in incorporating exercise training into the battery of therapeutic approaches for managing walking impairment in persons living with MS.

\section{Walking impairments in MS}

Walking impairment is one of the most life-limiting and common consequences of MS. The impairment in ambulation among persons with MS has been documented using numerous clinical, performance, physiological, and kinematic measures. The expanded disability status scale (EDSS) is the most commonly used measure in clinical research involving persons with $\mathrm{MS}^{7}$ and is heavily weighted by walking ability, particularly in the mid-range of scores (4.0-7.0), as measured by the distance walked over $500 \mathrm{~m} .{ }^{8}$ Natural history researchers have commonly defined benchmarks of disability in MS based on attainment of EDSS scores of 4.0 (ie, limited walking ability but able to walk more than $500 \mathrm{~m}$ without aid or rest) and 6.0 (ie, ability to walk with unilateral support no more than $100 \mathrm{~m}$ without rest); 9,10 those EDSS scores reflect the onset and progression of significant limitations in walking, respectively. To that end, $50 \%$ of persons with MS will reach the two benchmarks of walking impairment within 10-20 years of disease onset. ${ }^{9,10}$

Beyond the $500 \mathrm{~m}$ walk of the EDSS, researchers have often included other ambulatory performance measures for quantifying walking impairment in persons with MS; two of the most common are the timed 25 -foot walk (T25FW) ${ }^{11}$ and 6 -minute walk $(6 \mathrm{MW})^{12}$ tests. The T25FW measures speed of walking, whereas the $6 \mathrm{MW}$ measures distance traveled as an estimate of walking endurance. Both measures have demonstrated reduced performance as a function of MS disease status. For example, one study reported that $6 \mathrm{MW}$ distance was reduced in persons with MS versus controls, and 6MW distance was reduced as a function of increasing disability and self-reported ambulatory impairment (Multiple Sclerosis Walking Scale-12) within those who had MS. ${ }^{12}$ The existing cross-sectional research suggests that as MS-related disability progresses, persons with MS have associated deteriorations in walking speed and endurance.

Other researchers have included physiological measures for quantifying walking impairment in persons with MS such as the energetic or oxygen $\left(\mathrm{O}_{2}\right)$ cost of walking. The $\mathrm{O}_{2}$ cost of walking is measured in milliliters of $\mathrm{O}_{2}$ consumed per kilogram of bodyweight per meter travelled $\left(\mathrm{mL} \cdot \mathrm{kg}^{-1} \cdot \mathrm{m}^{-1}\right)$ and reflects the person's capacity to supply requisite energy for walking (ie, energetic efficiency of walking). To date, researchers have reported that the $\mathrm{O}_{2}$ cost of walking on a treadmill is elevated in persons with MS who have moderate disability compared with controls. ${ }^{13}$ We recently reported that the $\mathrm{O}_{2}$ cost of walking was significantly higher in persons with MS who had mild disability compared with controls and that disability scores were strongly associated with the $\mathrm{O}_{2}$ cost of different speeds of treadmill and over-ground walking. ${ }^{14}$ We further reported that $\mathrm{O}_{2}$ cost of walking was strongly correlated with Multiple Sclerosis Walking Scale-12 scores in persons with MS. ${ }^{15}$ This indicates that persons with MS require greater energy for ambulation and, by extension, walk less efficiently (ie, walking impairment); this inefficiency might reflect physiological or mechanical alterations in efficiency.

Some researchers have focused on spatial and temporal parameters of the gait cycle (ie, kinematic measures) for documenting walking impairment in MS. Such parameters describe alterations in the walking process itself and are important targets of gait rehabilitation. Researchers have reported that gait abnormalities such as reduced speed and stride length and prolonged double limb support time during walking are present even in persons with mild MS compared with matched controls. ${ }^{6}$ Other researchers have reported that speed, cadence, and stride length were dramatically reduced in a sample of persons with MS compared with matched controls, and the reduction was most pronounced in persons with MS who used a cane for assistance while walking. ${ }^{16}$ 
This indicates that parameters of the walking cycle itself are affected in persons with MS.

Collectively, the research clearly demonstrates a prevalent and significant degree of walking impairment in persons with MS. Walking impairment itself can be measured using a breadth of tools including clinical rating scales, performance and physiological markers, and alterations in spatial and temporal gait parameters. One source of walking impairment in MS is obviously associated with neurological injury and damage itself and thus progresses as the disease progresses. There are other factors that are associated with walking impairments such as physiological deconditioning in persons without MS. This underscores the importance of considering these other, nondisease factors that might be associated with walking function in MS. Such consideration, in turn, sets the stage for examining exercise training and physical activity as influences of walking impairment in MS.

\section{Physiological deconditioning, walking impairment, and $M S$}

Physiological deconditioning might influence the onset and progression of walking impairment in persons with chronic diseases,${ }^{17}$ including MS. ${ }^{18}$ Conceptually, MS is associated with a decrease in physical activity, which, in turn, can result in deconditioning across multiple domains of physiological functioning (eg, aerobic capacity, muscle strength, and balance). The resulting deconditioning feeds back and further drives physical inactivity in a cycle (Stage 1), until a threshold is reached that is likely to be associated with an impact on mobility. This then starts a cycle for the progression of walking impairment in MS (Stage 2), which might not entirely be linked with the burden of neurodegeneration in the CNS. This two-stage process is illustrated in Figure 1.

Empirically, there is strong evidence for general physical inactivity ${ }^{19}$ and physiological deconditioning ${ }^{18,20}$ in persons with MS. The physiological deconditioning manifests as reductions in aerobic capacity, muscle strength, and balance. ${ }^{20}$ There is initial evidence that physical inactivity and/or the disease itself are associated with reduced peak aerobic capacity ${ }^{21}$ as well as reduced isometric and voluntary force production, muscle cross-sectional area, type-I muscle fiber number, and biochemical function ${ }^{22,23}$ in persons with MS. Moreover, parameters of physiological functioning (eg, aerobic capacity and muscle strength) are important influences of mobility disability in senescence, ${ }^{24,25}$ $\mathrm{MS},{ }^{16,21}$ and other neurological conditions. ${ }^{26}$ The aforementioned conceptual model combined with empirical evidence support exercise training as an approach for attenuating the progression of walking impairment by increasing physical activity and improving physiological functioning in persons with MS. This is tantamount to preventing or disrupting the aforementioned cycle of inactivity and mobility impairment before or after its onset.

Importantly, there are other possible explanations for an effect of exercise training on walking impairment in MS. Exercise training might work via physiological functioning independently or in combination with effects on

\section{$\underline{\text { Stage } 1}$}

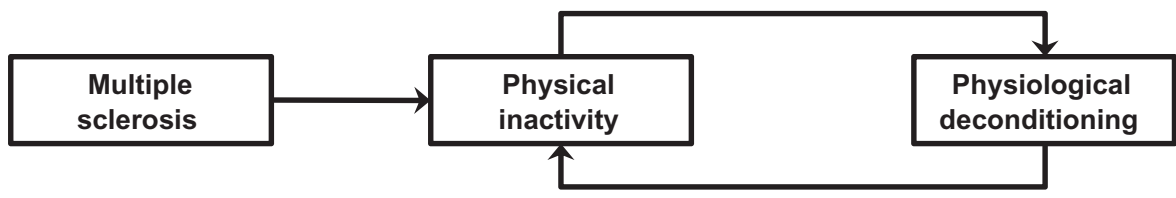

\section{Stage 2}

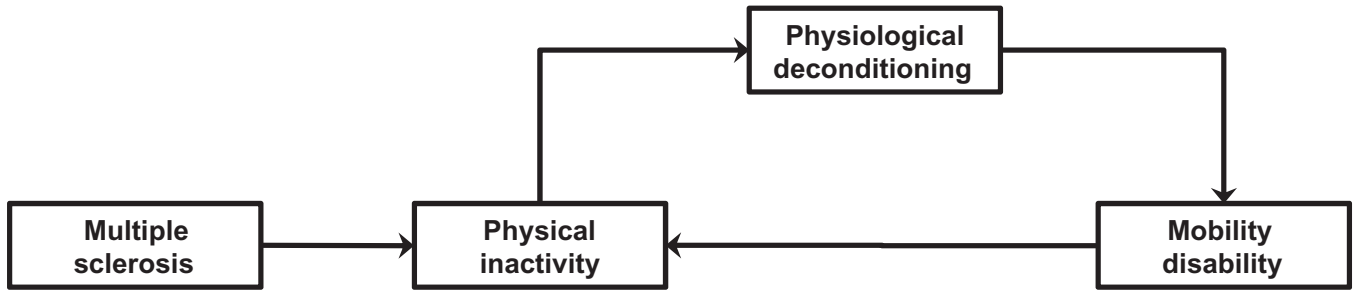

Figure I The two-stage cycle of physical inactivity, physiological deconditioning, and walking impairment in persons with MS. 
synaptogenesi ${ }^{27}$ and/or nerve growth factors. ${ }^{18}$ An additional possibility is that exercise training might have beneficial effects on cognition, fatigue, and mood as influences of walking impairment in MS.

\section{Exercise training, walking impairment, and MS}

Scientific evidence indicates that exercise training and physical activity might have beneficial effects on a number of outcomes (eg, fitness, mood, and quality of life), including walking impairment in MS. By definition, exercise is one component of physical activity that is planned, structured, and repetitive; performed over an extended period (ie, exercise training); and often prescribed by a physician or exercise specialist with a specific external objective such as improved fitness or health. ${ }^{28}$ Physical activity, by comparison, is defined as any bodily movement produced by contraction of skeletal muscles and that increases energy expenditure above resting levels. ${ }^{28}$ This broadly defined behavior includes exercise along with leisure-time physical activity, sport, occupational work, transportation, and household chores. By definition, exercise and physical activity are inter-related, but not synonymous, and available research demonstrates that both might impact walking impairment and MS-related disability.

Exercise training and physical activity are promising behavior strategies for managing many of the consequences associated with MS. The benefits have been summarized in general literature reviews, ${ }^{18,20,29}$ a systematic Cochran review, ${ }^{30}$ and a meta-analysis. ${ }^{31}$ One meta-analysis indicated that physical activity, in the form of exercise training, was associated with improved quality of life and fatigue in persons with MS. ${ }^{31}$ Other benefits of physical activity in persons with MS include improved muscle strength, body composition, and cardiorespiratory, and fitness as well as management of symptoms such as anxiety and depression. ${ }^{18,20,29,30}$

Of central importance for this paper, we have focused on the relationships among physical activity, exercise training, and markers of walking impairment in persons with MS. One of our studies examined the association between physical activity and walking impairment in a validation of physical activity measures among persons with MS. ${ }^{32}$ Thirty persons with MS wore a pedometer and a single-axis accelerometer over a 7-day period, completed the Godin Leisure-Time Exercise Questionnaire, and underwent a 7-day physical activity recall and an EDSS assessment for measuring mobility disability. Importantly, there were statistically significant and medium sized correlations between EDSS scores and physical activity as measured by total daily activity counts from the accelerometer $(r=-0.37, P<0.01)$ and total daily step counts from the pedometer $(r=-0.34, P<0.01)$. These results provide preliminary evidence of an association between objectively measured physical activity and mobility disability in a small sample of ambulatory persons with MS.

Another study examined the association between accelerometer activity counts as an objective measure of physical activity and scores from the Multiple Sclerosis Walking Scale-12. ${ }^{33}$ This scale is a 12 -item patient-rated outcome for measuring the impact of MS on walking (ie, mobility disability) that has been validated and included in clinical trials involving persons with MS. The sample of persons with MS $(N=133)$ was recruited through support group meetings of Midwestern chapters of the National MS Society. The participants completed the Multiple Sclerosis Walking Scale-12 along with other measures, including the EDSS, and wore a single-axis accelerometer over a 7-day period. There were statistically significant correlations between total daily activity counts from the accelerometry and Multiple Sclerosis Walking Scale-12 scores in the overall sample $(r=-0.64, P<0.01)$ and in the subsamples of persons with mild-to-moderate (EDSS scores of $1-4.5 ; r=-0.51$, $P<0.01$ ) and moderate-to-severe (EDSS scores of 5-8; $r=-0.48, P<0.01)$ MS-related disability. These results provide supplementary evidence of an association between objectively measured physical activity and mobility disability in MS, particularly among those with significant MS-related disability.

The next study involved a secondary analysis of data from an ongoing, longitudinal study of symptoms and physical activity $^{34}$ and focused on the association between physical activity and walking impairment in a large cohort of persons with MS $(N=269) .{ }^{35}$ The participants wore a single-axis accelerometer over a 7-day period and completed the Godin Leisure-Time Exercise Questionnaire, International Physical Activity Questionnaire, Multiple Sclerosis Walking Scale-12, and Patient-Determined Disease Steps. Bivariate correlation and confirmatory factor analyses indicated that (a) Godin Leisure-Time Exercise Questionnaire and International Physical Activity Questionnaire scores were strongly correlated and loaded significantly on a physical activity factor, (b) Multiple Sclerosis Walking Scale-12 and Patient-Determined Disease Steps scores strongly correlated and loaded significantly on a walking mobility factor, and (c) there was a moderate and statistically significant association between the physical activity and walking mobility factors $(r=-0.40, P<0.01)$. Such data further indicate that physical activity is associated 
with mobility disability but in a large sample of persons with MS using advanced statistical methodology.

The previously reviewed studies have been crosssectional and limit inferences about change. Using a panel design, we next examined physical activity as a predictor of disability progression in persons with MS over a 6-month period. ${ }^{36}$ Importantly, panel designs involve the collection of observations or data at more than one point in time using the same persons, and this design allows for studying the dynamics of relationship changes over time. Panel analysis, in turn, is an appropriate analytic procedure for testing hypothetical relationships about the effects of changes in variables on one another over time. The sample $(N=269)$ of persons with MS completed the Godin Leisure-Time Exercise Questionnaire, International Physical Activity Questionnaire, Multiple Sclerosis Walking Scale-12, and Patient-Determined Disease Steps before and after a 6-month observational period. The panel analysis indicated that baseline physical activity had a direct effect on walking impairment (path coefficient $=-0.31$ ), and change in physical activity across a 6-month period had a direct effect on residual change in progression of walking impairment (path coefficient $=-0.16$ ). This study was limited by the lack of performance measures of ambulation, but the findings provide preliminary support for a reduction in physical activity as a predictor of walking impairment over time in persons with MS. By extension, the delivery of an intervention that increases physical activity through exercise training or principles of behavior change could forestall, stabilize, or improve walking impairment in MS.

We have recently examined the overall effect of exercise training on walking mobility among persons with MS using meta-analytic procedures. ${ }^{37}$ This involved an extensive search for published exercise training studies. Studies were selected if they measured walking mobility, using instruments identified as acceptable walking mobility constructs and outcome measures for persons with neurological disorders (eg, EDSS, T25FW, 6MW, $\mathrm{O}_{2}$ cost of walking, Multiple Sclerosis Walking Scale-12, and temporal and spatial parameters from motion capture), before and after an intervention that included exercise training. We excluded published studies that only included physical therapy or exercise training as a small part of a physiotherapy program. This allowed for a focus on exercise training effects on walking outcomes. Of the 43 published papers we located and reviewed, only 22 provided enough data to compute effect sizes expressed as Cohen's $d$. We retrieved 66 effect sizes from the 22 publications, which included 609 MS participants, and the weighted mean effect size was $g=0.19(95 \%$ confidence interval $=0.09$, 0.28 ). This effect size is obviously small by conventional standards. Nevertheless, the effect size might have clinical relevance when compared with the overall effectiveness of disease modifying agents for reducing disease progression and EDSS scores $(d=0.20) .{ }^{38}$ The cumulative evidence from the meta-analysis supports that exercise training is associated with a small, but potentially meaningful, improvement in walking mobility among persons with MS.

Overall, the accumulating evidence supports the argument that increased physical activity and exercise training will have beneficial effects on walking function in persons with MS. This is based on cross-sectional, longitudinal, and experimental research designs that included diversity in the breadth of measures of walking and persons with MS (eg, clinical MS course, disease duration, and disability status) and a compendium of interventions (ie, exercise training and physical activity level changes). This research serves as a platform that can be used to expand and broaden our application of exercise training and physical activity as a therapeutic approach for managing mobility impairment in MS. This research further lays the groundwork for subsequent examinations of physiological functioning as a mechanism for improvements in walking impairment and the consideration of exercise training and pharmacological agents as a combinatory approach for addressing mobility impairment in MS.

\section{Future research on exercise training and walking impairment in MS: four agenda items}

As previously posited and illustrated in Figure 1, physiological deconditioning often results from physical inactivity that is common in MS. Physiological deconditioning may prove to be an important and reversible contributor to mobility impairment, over and beyond the disease itself, in persons with MS. ${ }^{17,18}$ To that end, exercise training and behavior change interventions are primary approaches for increasing structured and lifestyle physical activity, respectively, and, in turn, improving physiological functioning and slowing, stopping, or reversing mobility disability in those with MS. To date, there have been few randomized controlled trials to test this mechanistic hypothesis. Indeed, this is an area of research that is still in infancy and that holds promise to provide clinicians and therapists with an evidence-based model that guides the integration of exercise training and behavior change interventions into regimens for managing the progression of walking dysfunction in MS. Yet, many elements still 
to be understood include the optimal type or mode, intensity, frequency, duration, and maintenance of exercise training for optimizing physiological functioning and walking function in MS. One particular consideration is the optimal intensity of the exercise training stimulus. Indeed, exercise that is too intense might result in immune and stress hormone changes that are counter productive for beneficial effects on mobility outcomes and the MS disease course. We further note that too intense an exercise training stimulus might exacerbate and/or worsen MS-related fatigue. This is arguably one of the most impairing symptoms of MS. The worsening of fatigue might undermine long-term involvement in physical activity and/or exercise training.

Future research might consider examining the combinatory effects of exercise training plus pharmacological agents on walking mobility in MS. Perhaps the most obvious direction involves examining the combined, and perhaps synergistic, effects of exercise training plus the 4-aminopyridine fampradine (Ampyra $\AA$ ) on walking outcomes. The rationale for fampridine, in particular, is based on demyelinated axons having abnormal potassium currents that manifest in conduction failure by decreasing the propagation of action potentials. The potassium channel blocker 4-aminopyridine or fampradine should improve nerve impulse conduction and affect a variety of outcomes. There is evidence that fampridine improves visual function, strength, ambulation, fatigue, and endurance in persons with MS. ${ }^{39-42}$ The most exciting evidence for beneficial effects is from a randomized, multicentre, double-blind, controlled Phase III trial of 301 patients who were treated with either fampridine $(10 \mathrm{mg}$ twice daily; $\mathrm{n}=229)$ or placebo $(\mathrm{n}=72) .{ }^{43}$ The primary outcome was a responder analysis based on consistent improvement in the $\mathrm{T} 25 \mathrm{FW}$. The proportion of $\mathrm{T} 25 \mathrm{FW}$ responders was significantly higher in the fampridine group $(78 / 224$ or $35 \%)$ than in the placebo group $(6 / 72$ or $8 \%)$, indicating that fampridine was associated with an improvement in walking ability among persons with MS. The combination of exercise training and fampradine potentially has clinically meaningful, and perhaps synergistic, effects on mobility outcomes. There are two potential mechanisms for such augmentation. One is that exercise training and fampradine likely work through different mechanisms for improving mobility outcomes. The second is that fampradine might improve one's capacity for engaging in exercise training. This might allow for undertaking a greater exercise training stimulus and perhaps experiencing larger adaptations, including improvements in physiological functioning and reductions in walking impairment.
One additional application of exercise training might be for better tolerating the side effects of pharmacological agents commonly used in modifying the course of MS. Indeed, interferon-beta medications are first-line treatments for MS, with increasing evidence for short-term and long-term effects on the progression of mobility disability. ${ }^{38}$ One factor that undermines the long-term administration of such disease modifying agents is the occurrence of side effects such as flu-like symptoms and, in some cases, fatigue. To that end, research might consider the addition of exercise training as an approach for minimizing the side effects of interferonbeta medications, particularly fatigue. ${ }^{31}$ This would ideally allow for improved tolerance, adherence, and long-term outcomes.

The final consideration involves increased basic science research that examines effects of exercise training on MS using experimental autoimmune encephalomyelitis (EAE) as a model of MS. Indeed, researchers have recently provided exciting results regarding the effects of voluntary wheel running on clinical, synaptic, and neuropathological outcomes using EAE as a model of MS. ${ }^{27}$ This research suggests that lifestyle physical activity might play a central role in the clinical course of MS, and this basic science approach might ultimately identify that changes in the CNS account for improved ambulatory function with exercise training.

\section{Conclusion}

The adoption of exercise training and physical activity as behavioral influences of walking impairment in persons with MS might have considerable significance for public health and clinical care. Disease modifying agents have limited efficacy in slowing eventual progression of mobility disability over time, ${ }^{44,45}$ and researchers have argued for the identification of alternative approaches that retard the progression of mobility disability in persons with MS.9,10 We argue that exercise training and physical activity behavior change interventions might represent powerful, alternative approaches for mitigating the progression of walking impairment by improving physiological functioning in persons with MS. An alternative perspective is that the effect of exercise training and physical activity on walking might occur through synaptogenesis, nerve growth factors, or symptom management. Nevertheless, the relevance for clinicians and therapists who care for MS patients is that exercise training and physical activity behavior change interventions can be integrated within rehabilitation regimens for possibly preventing the onset and progression of mobility disability. This is consistent with the recommendation by some experts that rehabilitation 
is the only practical means of improving function, and perhaps attenuating the progression of walking impairment, in persons with MS. ${ }^{46}$ Future research on exercise training and physical activity has the potential to significantly advance the management of progressive walking impairment in MS, and this effect might be further examined in the context of disease modifying agents that affect walking ability and/or disease progression.

\section{Acknowledgment}

Robert W Motl was supported by a grant from the National Multiple Sclerosis Society (RG 3926A2/1) that facilitated the writing of this paper.

\section{Disclosure}

The authors report no conflicts of interest in this work.

\section{References}

1. Mayr WT, Pittock SJ, McClelland RL, Jorgernsen NW, Noseworthy JH, Rodriquez M. Incidence and prevalence of multiple sclerosis in Olmsted County, Minnesota, 1985-2000. Neurology. 2003;61:1373-1377.

2. National Multiple Sclerosis Society. Multiple Sclerosis Information Sourcebook. New York, NY: Information Resource Center and Library of the National Multiple Sclerosis Society; 2005.

3. Hemmer B, Nessler S, Zhou D, Kieseier B, Hartung H-P. Immunopathogenesis and immunotherapy of multiple sclerosis. Nat Clin Pract Neurol. 2006;2:201-211.

4. Trapp BD, Nave K-A. Multiple sclerosis: an immune or neurodegenerative disorder? Ann Rev Neurosci. 2008;31:247-269.

5. Bjartmar C, Trapp BD. Axonal and neuronal degeneration in multiple sclerosis: mechanisms and functional consequences. Curr Opin Neurol. 2001;14:271-278.

6. Martin CL, Phillips BA, Kilpatrick TJ, et al. Gait and balance impairment in early multiple sclerosis in the absence of clinical disability. Mult Scler. 2006;12:620-628.

7. Kurtzke JF. Historical and clinical perspectives of the Expanded Disability Status Scale. Neuroepidemiology. 2008;31:1-9.

8. Goldman MD, Motl RW, Rudick RA. Possible clinical outcome measures for clinical trials in patients with multiple sclerosis. Ther Adv Neurol Disord. 2010;3:229-239.

9. Confavreux C, Vukusic S, Adeline P. Early clinical predictors and progression of irreversible disability in multiple sclerosis: an amnesic process. Brain. 2003;126:770-782.

10. Confavreux C, Vukusic S, Moreau T, Adeleine P. Relapses and progression of disability in multiple sclerosis. $N$ Eng J Med. 2000;343: 1430-1438.

11. Schwid SR, Goodman AD, Mattson DH, et al. The measurement of ambulatory impairment in multiple sclerosis. Neurology. 1997;49(5): 1419-1424.

12. Goldman MD, Marrie RA, Cohen JA. Evaluation of the six-minute walk in multiple sclerosis subjects and healthy controls. Mult Scler. 2008; 14(3):383-390.

13. Olgiati R, Jacquet J, di Prampero PE. Energy cost of walking and exertional dypsnea in multiple sclerosis. Am Rev Respir Dis. 1986;134: 1005-1010.

14. Motl RW, Suh Y, Dlugonski D, et al. Oxygen cost of treadmill and over-ground walking in mildly disabled persons with multiple sclerosis. Neurol Sci. Epub 2010 Aug 27.

15. Motl RW, Dlugonski D, SuhY, et al. Multiple Sclerosis Walking Scale-12 and oxygen cost of walking. Gait Posture. 2010;31:506-510.
16. Thoumie P, Lamotte D, Cantalloube S, Faucher M, Amarenco G. Motor determinants of gait in 100 ambulatory patients with multiple sclerosis. Mult Scler. 2005;11:485-491.

17. Durstine JL, Painter P, Franklin BA, Morgan D, Pitetti KH, Roberts SO Physical activity for the chronically ill and disabled. Sports Med. 2000; 30:207-219.

18. White LJ, Castellano V. Exercise and brain health - Implications for multiple sclerosis. Part I - Neuronal growth factors. Sports Med. 2008;38: 91-100.

19. Motl RW, McAuley E, Snook EM. Physical activity and multiple sclerosis: a meta analysis. Mult Scler. 2005;11:459-463.

20. Garrett M, Coote S. Multiple sclerosis and exercise in people with minimal gait impairment - a review. Phys Ther Rev. 2009;14:169-180.

21. Motl RW, Goldman MD. Physical inactivity, neurological disability, and cardiorespiratory fitness in multiple sclerosis. Acta Neurol Scand. In press 2010.

22. Kent-Braun JA, Ng AV, Castro M, et al. Strength, skeletal muscle composition, and enzyme activity in multiple sclerosis. J Appl Physiol. 1997;83:1998-2004.

23. de Haan A, de Ruiter CJ, van der Woude LHV, Jongen PJH. Contractile properties and fatigue of quadriceps muscles in multiple sclerosis. Muscle Nerve. 2000;23:1534-1541.

24. Heckman GA, McKelvie RS. Cardiovascular aging and exercise in healthy adults. Clin J Sports Med. 2008;18:479-485.

25. Ploutz-Snyder LL, Manini T, Ploutz-Snyder RJ, Wolf DA. Functionally relevant thresholds of quadriceps femoris strength. J Gerontol A Biol Sci Med Sci. 2002;57:B144-B152.

26. Waters S, Mulroy S. The energy expenditure of normal and pathological gait. Gait Posture. 1999;9:207-231.

27. Rossi S, Furlan R, de Chiara V, et al. Exercise attenuates the clinical, synaptic and dendritic abnormalities of experimental autoimmune encephalomyelitis. Neurobiol Dis. 2008;36:51-59.

28. Bouchard C, Shephard RJ. Physical activity, fitness, and health: the model and key concepts. In: Bouchard C, Shephard RJ, Stephens T, editors. Physical Activity, Fitness, and Health: International Proceedings and Consensus Statement. Champaign, IL: Human Kinetics; 1994:77-88.

29. White LJ, Dressendorfer RH. Exercise and multiple sclerosis. Sports Med. 2004;34:1077-1100.

30. Rietberg MB, Brooks D, Uitdehaag BMJ, Kwakkel G. Exercise therapy for multiple sclerosis. Cochrane Database System Review. 2004.

31. Motl RW, Gosney JL. Effect of exercise training on quality of life in multiple sclerosis: a meta-analysis. Mult Scler. 2008;14:129-135.

32. Motl RW, McAuley E, Snook EM, Scott JA. Validity of physical activity measures in ambulatory individuals with multiple sclerosis. Disabil Rehabil. 2006;28:1151-1156.

33. Motl RW, Snook EM. Confirmation and extension of the validity of the Multiple Sclerosis Walking Scale-12 (MSWS-12). J Neurol Sci. 2008; 268:69-73.

34. Weikert M, Motl RW, Suh Y, McAuley E, Wynn D. Accelerometry in persons with multiple sclerosis: Measurement of physical activity or walking mobility? J Neurol Sci. 2010;290:6-11.

35. Motl RW, McAuley E, Wynn D, Suh Y, Weikert M, Dlugonski D. Symptoms and physical activity among adults with relapsing-remitting multiple sclerosis? J Nerv Ment Dis. 2010;198:213-219.

36. Motl RW, McAuley E, Wynn D, Vollmer T. Lifestyle physical activity and walking impairment over time in relapsing-remitting multiple sclerosis: results from a panel study. Am J Phys Med Rehabil. In press 2010.

37. Snook EM, Motl RW. Effect of exercise training on walking mobility in multiple sclerosis: a meta-analysis. Neurorehabil Neural Repair. 2009;23:108-116.

38. Filippini G, Munari L, Incorvaia B, et al. Interferons in relapsing remitting multiple sclerosis: a systematic review. Lancet. 2003;361:545-552.

39. van Diemen HA, Polman CH, van Dongen TM, et al. The effect of 4-aminopyridine on clinical signs in multiple sclerosis: a randomized, placebo-controlled, double-blind, cross-over study. Ann Neurol. 1992; $32: 123-130$ 
40. Bever CT Jr, Young D, Anderson PA, et al. The effects of 4-aminopyridine in multiple sclerosis patients: results of a randomized, placebo-controlled, double-blind, concentration-controlled, crossover trial. Neurology. 1994;44:1054-1059.

41. Schwid SR, Petrie MD, McDermott MP, Tierney DS, Mason DH, Goodman AD. Quantitative assessment of sustained-release 4-aminopyridine for symptomatic treatment of multiple sclerosis. Neurology. 1997;48:817-821.

42. Stefoski D, Davis FA, Fitzsimmons WE, Luskin SS, Rush J, Parkhurst GW. 4-Aminopyridine in multiple sclerosis: prolonged administration. Neurology. 1991;41:1344-1348.
43. Goodman AD, Brown TR, Krupp LB, et al. Sustained-release oral fampridine in multiple sclerosis: a randomised, double-blind, controlled trial. Lancet. 2009;373(9665):732-738.

44. Katrych O, Simone TM, Azad S, Mousa SA. Disease-modifying agents in the treatment of multiple sclerosis: a review of long-term outcomes. CNS Neurol Disord Drug Targets. 2009;8:512-519.

45. Khan O, Leist TP, Vollmer TL, Zamvil SS. Investigating multiple sclerosis: targeting therapeutic options. Int J MS Care. 2008; 10(Suppl):5-20.

46. Kraft GH. Rehabilitation still the only way to improve function in multiple sclerosis. Lancet. 1999;354:2016-2017.

\section{Publish your work in this journal}

Neuropsychiatric Disease and Treatment is an international, peerreviewed journal of clinical therapeutics and pharmacology focusing on concise rapid reporting of clinical or pre-clinical studies on a range of neuropsychiatric and neurological disorders. This journal is indexed on PubMed Central, the 'PsycINFO' database and CAS, and is the official journal of The International Neuropsychiatric Association (INA). The manuscript management system is completely online and includes a very quick and fair peer-review system, which is all easy to use. Visit http://www.dovepress.com/testimonials.php to read real quotes from published authors.

Submit your manuscript here: http://www.dovepress.com/neuropsychiatric-disease-and-treatment-journal 